PROCEEDINGS OF THE

AMERICAN MATHEMATICAL SOCIETY

Volume 133, Number 6, Pages 1851-1859

S 0002-9939(05)07743-9

Article electronically published on January 21, 2005

\title{
BOREL CLASSES OF SETS OF EXTREME AND EXPOSED POINTS IN $\mathbb{R}^{n}$
}

\author{
PETR HOLICKÝ AND TAMÁS KELETI \\ (Communicated by Carl G. Jockusch, Jr.)
}

\begin{abstract}
It is known that the sets of extreme and exposed points of a convex Borel subset of $\mathbb{R}^{n}$ are Borel. We show that for $n \geq 4$ there exist convex $G_{\delta}$ subsets of $\mathbb{R}^{n}$ such that the sets of their extreme and exposed points coincide and are of arbitrarily high Borel class. On the other hand, we show that the sets of extreme and of exposed points of a convex set $C \subset \mathbb{R}^{3}$ of additive Borel class $\alpha$ are of ambiguous Borel class $\alpha+1$. For proving the latter-mentioned results we show that the union of the open and the union of the closed segments of $C \cap \partial C$ are of the additive Borel class $\alpha$ if $C \subset \mathbb{R}^{3}$ is a convex set of additive Borel class $\alpha$.
\end{abstract}

\section{INTRODUCTION}

Rogers showed in 9 that the set $\operatorname{ext} C$ of all extreme points of every convex Borel subset of $\mathbb{R}^{n}$ is Borel. Jayne and Rogers proved in the introduction of [5] that also the set $\exp C$ of all exposed points of any convex Borel subset of $\mathbb{R}^{n}$ is Borel. Thus they improved the result of [1] that $\exp C$ is Borel if $C$ is a closed convex subset of a Euclidean space. The mentioned results and the methods of their proofs in [9] and [5] do not give any information about the dependence of the Borel classes of the sets $\operatorname{ext} C$ and $\exp C$ on the Borel class of $C$. The only published results in this direction that we know about concern convex $F_{\sigma}$ subsets of $\mathbb{R}^{n}$. Jayne and Rogers explain in $\left[5\right.$ p. 252] that even for every convex $K_{\sigma}$ subset $C$ of any Banach space, the set ext $C$ is $G_{\delta}$ in $C$. Choquet, Corson and Klee give a more detailed description of $\exp C$ for closed convex subsets of $\mathbb{R}^{n}$ in [1, Theorem 1.1] depending on the dimension $n$. For arbitrary $n$ their result gives that $\exp C$ is of ambiguous class $\Delta_{3}^{0}$. Theorem 1 and the remark "Added in proof" at the end of Section 1 in [3] give in particular that $\exp C$ is in $\Pi_{3}^{0}$ for every convex $K_{\sigma}$ subset of a Banach space.

Received by the editors February 10, 2003 and, in revised form, August 23, 2003 and February $29,2004$.

2000 Mathematics Subject Classification. Primary 03E15, 28A05, 52A20, 52A15.

Key words and phrases. Convex set, extreme point, exposed point, Borel class.

The first author was supported by the "Mathematics in Information Society" project carried out by the Alfréd Rényi Institute of Mathematics - Hungarian Academy of Sciences, in the framework of the European Community's "Confirming the International Role of Community Research" program. The research was partly supported also by grants GAČR 201/03/0931 and MSM 113200007.

The second author was supported by OTKA grants F 029768 and F 043620. 
In Example 3.1 we show that for convex $G_{\delta}$ subsets $C$ of $\mathbb{R}^{n}, n \geq 4$, one cannot get any uniform bound on the Borel classes of the sets of extreme and exposed points of $C$. On the other hand, we prove that in $\mathbb{R}^{3}$ there is a bound $\beta(\alpha)$ of the Borel classes of ext $C$ and $\exp C$ depending on the Borel class $\alpha$ of $C$ only. We also give examples showing that our estimates are sharp.

\section{Preliminaries}

For any two distinct points $a, b \in \mathbb{R}^{n}$ we use the notation $] a, b[$ for the open segment $\{(1-t) a+t b ; 0<t<1\}$ and $[a, b]$ for the closed segment $\{(1-t) a+t b ; 0 \leq$ $t \leq 1\}$.

Let us recall that a point $x$ of a convex set $C$ is called an extreme point of $C$ if $x \notin] a, b[$ for any distinct elements $a$ and $b$ of $C$.

The point $x$ is called an exposed point of a convex set $C$ if there is a continuous linear functional which attains its maximum on $C$ at $x$ and only at $x$. (Note that every exposed point of a convex set $C$ is also an extreme point of $C$, but the converse is not always true.)

As we already mentioned in the introduction, we use $\operatorname{ext} C$ and $\exp C$ to denote the sets of all extreme and exposed points of $C$, respectively.

Given a nontrivial linear form $L: \mathbb{R}^{n} \rightarrow \mathbb{R}$ and a real number $r$, we call the hyperplane $S=\left\{x \in \mathbb{R}^{n} ; L(x)=r\right\}$ a supporting hyperplane of a convex set $C$ (at point $x \in C$ ) if $L(x)=r$ and either $L(y) \leq r$ for all $y \in C$ or $L(y) \geq r$ for all $y \in C$. So $x$ is an exposed point of a convex set $C$ if and only if $\{x\}=C \cap S$ for some supporting hyperplane of $C$.

If $S$ is a supporting hyperplane of $C$ and the affine hull of $S \cap C$ has dimension $n$, we say that $S \cap C$ is an $n$-dimensional face of $C$. (Let us notice that our $n$ dimensional faces do not coincide with $n$-faces of [1].)

The boundary, the closure and the convex hull of a set $A$ will be denoted by $\partial A$, $\bar{A}$ and $\operatorname{conv} A$, respectively.

We use the notation $\Pi_{\alpha}^{0}, \Sigma_{\alpha}^{0}$ and $\Delta_{\alpha}^{0}$ in $M$ for the multiplicative, additive and ambiguous classes $\Pi_{\alpha}^{0}(M), \Sigma_{\alpha}^{0}(M)$ and $\Delta_{\alpha}^{0}(M)$ of Borel subsets of the metric space $M$, respectively:

$\Pi_{1}^{0}(M)$ stands for the closed, $\Sigma_{1}^{0}(M)$ for the open and $\Delta_{1}^{0}(M)$ for the clopen subsets of $M$,

$$
\begin{aligned}
& \Sigma_{\alpha}^{0}(M)=\left\{\bigcup_{n \in \mathbb{N}} A_{n} ; A_{n} \in \Pi_{\alpha_{n}}^{0}(M), \alpha_{n}<\alpha\right\}, \\
& \Pi_{\alpha}^{0}(M)=\left\{M \backslash A ; A \in \Sigma_{\alpha}^{0}(M)\right\} \text { and } \\
& \Delta_{\alpha}^{0}(M)=\Pi_{\alpha}^{0}(M) \cap \Sigma_{\alpha}^{0}(M)
\end{aligned}
$$

for $1<\alpha<\omega_{1}$ (cf. [6] p. 68]). We omit $M$ if $M=\mathbb{R}^{n}$ and the value of $n$ is clear from the context. We preferably use the more descriptive notation $G_{\delta}$ for $\Pi_{2}^{0}, F_{\sigma}$ for $\Sigma_{2}^{0}$, etc.

\section{EXAmple IN $\mathbb{R}^{4}$}

The following example shows that, if $n \geq 4$, then $\operatorname{ext} C$ and $\exp C$ can belong to arbitrary exact Borel classes (except for $\Sigma_{1}^{0}$ ) even if $C$ is a convex $G_{\delta}$ subset of $\mathbb{R}^{n}$. One has to realize only that in any interval there are Borel subsets of all exact Borel classes [6, Theorem 22.4].

Example 3.1. For any Borel subset $E$ of a circle in $\mathbb{R}^{4}$ (e.g. $E \subset\left\{x \in \mathbb{R}^{4} ; x_{1}^{2}+\right.$ $\left.\left.x_{2}^{2}=1, x_{3}=x_{4}=0\right\}\right)$, there is a bounded convex $G_{\delta}$ set $C \subset \mathbb{R}^{4}$ such that $\operatorname{ext} C=\exp C=E$. 
Proof. There are obvious examples if $E=\emptyset$. Thus we may assume without loss of generality that $E \subset\left\{x \in \mathbb{R}^{4} ; x_{1}^{2}+x_{2}^{2}=1, x_{3}=x_{4}=0\right\}$ and $(1,0,0,0) \in E$. Denote $B=\{a \in[0,2 \pi) ;(\cos a, \sin a, 0,0) \notin E\}$. We have $0 \notin B$. By a classical theorem (see e.g. [6, p. 83]), there is a one-to-one continuous map $f: F \rightarrow B$ of a $G_{\delta}$ subset $F$ of $[0,1]$ onto the Borel set $B$. We denote the graph of $f$ by $G$ and note that it is a $G_{\delta}$ subset of $[0,1] \times[0,2 \pi)$ and the projection of $G$ onto $B$ is injective.

Now we define our set $C$ by the equality $C=C_{0} \cup C_{1} \cup C_{2}$, where

$$
\begin{aligned}
& C_{0}=\left\{(x, y, q, h) \in \mathbb{R}^{4} ; x^{2}+y^{2}<1, q \in(-1,1), h \in(-1,1)\right\}, \\
& C_{1}=\left\{(x, y, 0,0) \in \mathbb{R}^{4} ; x^{2}+y^{2}=1\right\} \text { and } \\
& C_{2}=\left\{(\cos a, \sin a, h p, h) \in \mathbb{R}^{4} ;(p, a) \in G, h \in(-1,1)\right\} .
\end{aligned}
$$

It is clear that the set $C$ is bounded and the sets $C_{0}$ and $C_{1}$ are $G_{\delta}$ sets. We shall use the well-known theorem that homeomorphic images of $G_{\delta}$ subsets of complete metric spaces are always $G_{\delta}$ sets (see e.g. [7, $\left.\left.\S 35, \mathrm{III}\right]\right)$. The set $S=\{(a, h p, h) \in$ $\left.\mathbb{R}^{3} ;(p, a) \in G, h \in(-1,0) \cup(0,1)\right\}$ being homeomorphic to $G \times((-1,0) \cup(0,1))$ is a $G_{\delta}$ set, too. Since the map taking each triple $(a, q, h) \in S$ to the quadruple $(\cos a, \sin a, q, h) \in C$ is a homeomorphism of $S$ onto $C_{2} \backslash C_{1}$ (because $a$ cannot be 0 since we assumed that $0 \notin B$ ), we conclude that $C=C_{0} \cup C_{1} \cup C_{2}$ is a $G_{\delta}$ subset of $\mathbb{R}^{4}$.

The set $C_{0}$ is obviously convex. The set $C$ contains $C_{0}$ as a subset and it is contained in its closure. Thus, for proving that $C$ is convex, it is enough to prove that any supporting hyperplane of $\overline{C_{0}}$ intersects $C$ in a convex set. By definition, $C$ consists of $C_{0}$, of open segments $\left\{(\cos a, \sin a, h p, h) \in \mathbb{R}^{4} ; h \in(-1,1)\right\}$ for $a \in B$ (one for each $a \in B$ since the projection of $G$ onto $B$ is injective) and of points $(\cos a, \sin a, 0,0)$ for $a \in[0,2 \pi) \backslash B$. For fixed $a \in \mathbb{R}$, at the points of $\partial C_{0}$ with fixed first coordinates $(\cos a, \sin a)$, the set $\overline{C_{0}}$ has the same supporting hyperplane and this hyperplane intersects $\overline{C_{0}}$ in the $(2$-dimensional $)$ plane $\{(\cos a, \sin a, q, h) \in$ $\left.\mathbb{R}^{4} ;(q, h) \in \mathbb{R}^{2}\right\}$. Since the intersection of each such plane with $C$ is either a segment or a point, $C$ is a convex set.

The facts mentioned in the previous paragraph give also that the only exposed points of $C$ are the points $(\cos a, \sin a, 0,0)$ for $a \in[0,2 \pi) \backslash B$, and thus $\exp C=$ $\operatorname{ext} C=E$.

\section{EXtreme AND EXPOSED POINTS IN $\mathbb{R}^{3}$}

In the sequel we shall prove the following two theorems.

Theorem 4.1. Let $C \subset \mathbb{R}^{3}$ be a convex set of Borel class $\Sigma_{\alpha}^{0}$. Then the set $\operatorname{ext} C$ is of Borel class $\Pi_{\alpha}^{0}$ in $C$.

Theorem 4.2. Let $C \subset \mathbb{R}^{3}$ be a convex set of Borel class $\Sigma_{\alpha}^{0}$.

If $\alpha \neq 2$, then the set $\exp C$ is of Borel class $\Pi_{\alpha}^{0}$ in $C$.

If $\alpha=2$, then $\exp C=(F \cap G) \cup H$, where $F$ is an $F_{\sigma}$ set in $\mathbb{R}^{3}, G$ is a $G_{\delta}$ set in $\mathbb{R}^{3}$ and $H$ is a $G_{\delta}$ set in $C$.

As an immediate corollary of these theorems we get the following result concerning Borel classes in $\mathbb{R}^{3}$.

Corollary 4.3. Let $C \subset \mathbb{R}^{3}$ be convex.

If $C$ belongs to the additive Borel class $\Sigma_{\alpha}^{0}$, then the sets $\operatorname{ext} C$ and $\exp C$ are of ambiguous class $\Delta_{\alpha+1}^{0}$ in $\mathbb{R}^{3}$. 
If $C$ belongs to the ambiguous Borel class $\Delta_{\alpha}^{0}$, then the sets $\operatorname{ext} C$ (for any $\alpha$ ) and $\exp C($ for $\alpha \neq 2)$ are of multiplicative class $\Pi_{\alpha}^{0}$ in $\mathbb{R}^{3}$.

In particular, if $C$ belongs to the multiplicative Borel class $\Pi_{\alpha}^{0}$, then the sets $\operatorname{ext} C$ (for any $\alpha)$ and $\exp C($ for $\alpha \neq 1)$ are of multiplicative class $\Pi_{\alpha+1}^{0}$ in $\mathbb{R}^{3}$.

Remark 4.4. In the particular case when $C$ is closed (and more generally also if $C \in \Delta_{2}^{0}$ ), the set $H$ in Theorem 4.2 is $G_{\delta}$ also in $\mathbb{R}^{3}$ (since it is $G_{\delta}$ in a $G_{\delta}$ set) and we get the same description of $\exp C$ as Choquet, Corson and Klee in [1. Theorem 1.1(b)].

The most complicated description of $\exp C$ for an $F_{\sigma}$ set $C$ is necessary. An example of Corson [2] shows that there is a compact convex set $C \subset \mathbb{R}^{3}$ such that $\exp C$ is not the union of an $F_{\sigma}$ set and a $G_{\delta}$ set. Let us also notice that Holický and Laczkovich [4] observed recently that there is a compact convex set $C \subset \mathbb{R}^{3}$ such that $\exp C$ is not the intersection of an $F_{\sigma}$ set and a $G_{\delta}$ set.

Before coming to the proofs of both theorems, we illustrate in the next examples that there is not much space for improving the estimates of the classes of ext $C$ and $\exp C$ in Theorem 4.1, Theorem 4.2 and Corollary 4.3

Example 4.5. There is a convex set $D \subset \mathbb{R}^{3}$ of class $\Sigma_{\alpha}^{0}$ for $\alpha \geq 2$ such that $\operatorname{ext} D=\exp D$ is not $\Sigma_{\alpha}^{0}$ in $D$.

Proof. Let $B \subset S=\left\{(x, y) \in \mathbb{R}^{2} ; x^{2}+y^{2}=1\right\}$ be in $\Pi_{\alpha}^{0} \backslash \Sigma_{\alpha}^{0}, \alpha \geq 2$. Put

$$
D=\left(\left\{(x, y) \in \mathbb{R}^{2} ; x^{2}+y^{2}<1\right\} \times(-1,1)\right) \cup((S \backslash B) \times(-1,1)) \cup(S \times\{0\}) .
$$

Then $D$ is clearly convex and $\Sigma_{\alpha}^{0}$, but $\operatorname{ext} D=\exp D=B \times\{0\}$ is not $\Sigma_{\alpha}^{0}$ in $D$ (otherwise it would be in $\Sigma_{\alpha}^{0}$ in $\mathbb{R}^{3}$ because $S \times\{0\} \subset D$ is closed in $\mathbb{R}^{3}$ ).

Example 4.6. For any $\alpha \geq 1$, there is a convex $\Pi_{\alpha}^{0}$ set $E \subset \mathbb{R}^{3}$ with $\operatorname{ext} E=\exp E$ not $\Sigma_{\alpha+1}^{0}$ in $E$ and so not $\Sigma_{\alpha+1}^{0}$ in $\mathbb{R}^{3}$.

Proof. Let $S=\left\{(x, y) \in \mathbb{R}^{2} ; x^{2}+y^{2}=1\right\}$ and $F_{n}, n \in \mathbb{N}$, in $\Pi_{\alpha}^{0}(S)$ be such that their union $\bigcup_{n \in \mathbb{N}} F_{n}$ is not in $\Pi_{\alpha+1}^{0}(S)$. We may and shall suppose that $F_{n} \subset F_{n+1}$ for $n \in \mathbb{N}$.

If $\alpha=1$, we put

$$
E=\operatorname{conv}\left(\bigcup_{n \in \mathbb{N}}\left(F_{n} \times\left[-\frac{1}{n}, \frac{1}{n}\right]\right) \cup(S \times\{0\})\right) .
$$

Using the well-known fact that the convex hull of a compact set is compact (see e.g. in [8, Theorem 17.2]) we get that $E$ is compact. It is easy to check that $\operatorname{ext} E \cap S=\exp E \cap S=S \backslash \bigcup_{n \in \mathbb{N}} F_{n}$. Since $S \times\{0\} \subset E$ is closed and $\bigcup_{n \in \mathbb{N}} F_{n}$ is not $G_{\delta}$ in $S$, this implies that ext $E$ and $\exp E$ are not $F_{\sigma}$ in $E$. (Let us also note that the stronger examples of [2] or [4] also give the case $\alpha=1$ for exposed points.)

If $\alpha>1$, put

$$
E=\left(\left\{(x, y) \in \mathbb{R}^{2} ; x^{2}+y^{2}<1\right\} \times(-1,1)\right) \cup(S \times\{0\}) \cup \bigcup_{n \in \mathbb{N}}\left(F_{n} \times\left(-\frac{1}{n}, \frac{1}{n}\right)\right) .
$$

Let $\alpha_{k}=\alpha-1$ if $\alpha$ is a nonlimit ordinal, and let $\left(\alpha_{k}\right)$ be an increasing sequence of ordinals that converges to $\alpha$ if $\alpha$ is a limit ordinal. In both cases there exist sets 
$G_{k}^{n}$ in $\Sigma_{\alpha_{k}}^{0}(S)$ such that $F_{n}=\bigcap_{k \in \mathbb{N}} G_{k}^{n}$ and $G_{k+1}^{n} \subset G_{k}^{n}$ for $n, k \in \mathbb{N}$. Then one can easily check that

$$
\bigcup_{n \in \mathbb{N}} F_{n} \times\left(0, \frac{1}{n}\right)=\bigcap_{k \in \mathbb{N}} \bigcup_{n \in \mathbb{N}} G_{k}^{n} \times\left(\frac{1}{n+2}, \frac{1}{n}\right) .
$$

Hence $E$ is the union of $\left(\left\{(x, y) \in \mathbb{R}^{2} ; x^{2}+y^{2}<1\right\} \times(-1,1)\right) \cup(S \times\{0\})$ and

$$
\bigcap_{k \in \mathbb{N}} \bigcup_{n \in \mathbb{N}} G_{k}^{n} \times\left(\left(\frac{1}{n+2}, \frac{1}{n}\right) \cup\left(-\frac{1}{n},-\frac{1}{n+2}\right)\right),
$$

which implies that $E$ is a $\Pi_{\alpha}^{0}$ set. The set $E$ is clearly convex. However, ext $E=$ $\exp E=S \backslash \bigcup_{n \in \mathbb{N}} F_{n}$ is not $\Sigma_{\alpha+1}^{0}$ in $E$.

\section{Proof of Theorem 4.1}

Lemma 5.1. Let $C \subset \mathbb{R}^{3}$ be a convex set of Borel class $\Sigma_{\alpha}^{0}$, and $P_{0}, P_{1}$ be two distinct parallel planes in $\mathbb{R}^{3}$. Then the set

$$
S=\bigcup\{] a, b\left[;[a, b] \subset \partial C, a \in C \cap P_{0}, b \in C \cap P_{1}\right\}
$$

is of class $\Sigma_{\alpha}^{0}$, too.

Proof. Since the case $\alpha=1$ is trivial, we may suppose that $\alpha \geq 2$.

There are at most countably many maximal "quadrangles" $Q_{k}=\operatorname{conv}\left(J_{0} \cup J_{1}\right)$, where $J_{i} \subset \partial C \cap P_{i}, i=0,1$, are bounded or unbounded line segments. Let us denote by $G_{k}$ the relative interior of $Q_{k}$ in the affine span of $Q_{k}$ and put $G=\bigcup_{k} G_{k}$. Thus each $G_{k}$ is an $F_{\sigma}$ set in $\mathbb{R}^{3}$ and open in $\partial C$.

The intersection $S \cap G$ is the countable union of $S \cap G_{k}$, each of the form $\operatorname{conv}\left(I_{0} \cup I_{1}\right) \cap G_{k}$, where $I_{0} \subset J_{0}$ and $I_{1} \subset J_{1}$ are convex subsets of a line, and so $S \cap G$ is an $F_{\sigma}$ set.

The map taking each triple $\left(x_{0}, x_{1}, t\right) \in\left(\partial C \cap P_{0}\right) \times\left(\partial C \cap P_{1}\right) \times(0,1)$ to the point $(1-t) x_{0}+t x_{1} \in C$ is continuous. Let us denote by $f$ its restriction to the set

$$
K=\left\{\left(x_{0}, x_{1}, t\right) \in\left(\partial C \cap P_{0}\right) \times\left(\partial C \cap P_{1}\right) \times(0,1) ; \frac{x_{0}+x_{1}}{2} \in \partial C \backslash G\right\} .
$$

Note that if $] x_{0}, x_{1}\left[\cap \partial C \neq \emptyset\right.$ for some $\left(x_{0}, x_{1}\right) \in\left(\bar{C} \cap P_{0}\right) \times\left(\bar{C} \cap P_{1}\right)$, then $\left[x_{0}, x_{1}\right] \subset \partial C$.

This implies that

$$
f(K \cap(C \times C \times(0,1)))=S \backslash G .
$$

The previous observation also implies that, whenever two distinct open segments with endpoints in $\partial C \cap P_{0}$ and $\partial C \cap P_{1}$ have nonempty intersection in $\partial C$, then their midpoints belong to $G$. This proves that $f$ is injective.

The set $K$ can be written as the countable union of compact sets $K_{n}$. Each restriction $f \nmid K_{n}$ being continuous and injective on a compact set is a homeomorphism taking the $\Sigma_{\alpha}^{0}$ subset $K_{n} \cap(C \times C \times(0,1))$ of $K_{n}$ to a $\Sigma_{\alpha}^{0}$ set (in the compact set $f\left(K_{n}\right)$ and so in $\mathbb{R}^{3}$ as well) and thus $S \backslash G=\bigcup_{n} f\left(K_{n} \cap(C \times C \times(0,1))\right)$ is also a $\Sigma_{\alpha}^{0}$ set. 
Proof of Theorem 4.1. For $i \in\{1,2,3\}$ and a rational number $r \in \mathbb{Q}$, we put $P_{i}(r)=\left\{\left(x_{1}, x_{2}, x_{3}\right) \in \mathbb{R}^{3} ; x_{i}=r\right\}$. Clearly,

$$
\begin{gathered}
C \cap \partial C \backslash \operatorname{ext} C=\bigcup\{] a, b[;] a, b[\subset C \cap \partial C\} \\
=\bigcup_{i, p, q} \bigcup\{] a, b\left[;[a, b] \subset \partial C, a \in C \cap P_{i}(p), b \in C \cap P_{i}(q)\right\},
\end{gathered}
$$

where the union $\bigcup_{i, p, q}$ is over all triples $(i, p, q) \in\{1,2,3\} \times \mathbb{Q} \times \mathbb{Q}$ such that $p<q$. By Lemma 5.1 with $P_{0}=P_{i}(p)$ and $P_{1}=P_{i}(q)$, and by the countability of the index set $\{1,2,3\} \times \mathbb{Q} \times \mathbb{Q}$, the set $C \cap \partial C \backslash \operatorname{ext} C$ is $\Sigma_{\alpha}^{0}$ in $\mathbb{R}^{3}$ and thus $\operatorname{ext} C$ is $\Pi_{\alpha}^{0}$ in $C$.

\section{Proof of Theorem 4.2}

The next lemma enables us to reduce the investigation of the set of exposed points of convex sets $C \subset \mathbb{R}^{3}$ to the study of the union $U^{C}$ of the closed segments of $C \cap \partial C$. We get it by modifying a description indicated in the proof of [1. Theorem 1.1].

Lemma 6.1. Let $C$ be a convex subset of $\mathbb{R}^{3}$. Then

$$
\exp C=\left(C \cap \partial C \backslash U^{C}\right) \cup(E \cap C \backslash \bigcup\{I \cap C \backslash \exp C ; I \in \mathcal{I}\}),
$$

where

$$
\begin{gathered}
U^{C}=\bigcup\{[a, b] ;[a, b] \subset C \cap \partial C\}, \\
E=\{x ;\{x\}=S \cap[x, c] \text { for an }[x, c] \subset \partial C \text { and a supporting plane } S \text { of } \bar{C}\} \\
\text { and } \\
\qquad \begin{array}{l}
\mathcal{I}=\{I ; I=T \cap S \cap C, S, T \text { are distinct supporting planes of } \bar{C}, \\
\qquad \operatorname{dim} I=1, \operatorname{dim}(T \cap \bar{C})=2\} .
\end{array} \\
\text { Moreover, the sets } E \text { and } \bigcup\{I \cap C \backslash \exp C ; I \in \mathcal{I}\} \text { are } F_{\sigma} \text { sets in } \mathbb{R}^{3} .
\end{gathered}
$$

Proof. Let $x$ be an exposed point of $C$ which belongs to some segment $[a, b] \subset$ $C \cap \partial C$. Then $x \in E$ and thus the inclusion " $C$ " is proved.

For proving the other inclusion first note that $C \cap \partial C \backslash U^{C} \subset \exp C$ is clear. Thus it is enough to prove that $x \in \bigcup \mathcal{I}$ if $x \notin \exp C$ and $x \in E \cap C$.

So suppose now that $x \notin \exp C$ and $x \in E \cap C$. Then there is a supporting plane $S$ of $\bar{C}$ with $\{x\}=S \cap[x, t]$ for some $[x, t] \subset \partial C$. Since $x \notin \exp C$ there is an $s$ such that $[x, s] \subset S \cap C$. There is a supporting plane $T \supset[x, t]$ of $\bar{C}$ and since $t \notin S$ we have that $S \neq T$. The point $x$ belongs to the line $T \cap S$ and $T \cap S \cap C \neq\{x\}$ because otherwise $x$ would be an exposed point of $C$ (a suitable rotation $P$ of the plane $S$ around $S \cap T$ would be a supporting plane with $P \cap C=\{x\}$ ). So $I=S \cap T \cap C$ is a segment on the boundary of $C$. Moreover, $T \cap \bar{C}$ contains the two-dimensional set $\operatorname{conv}(I \cup\{t\})$, and so $x \in \bigcup \mathcal{I}$ and the equality is proved.

The family $\mathcal{I}$ is countable since the set of two-dimensional faces of $\bar{C}$ is countable and the set of one-dimensional faces of each two-dimensional face is also countable. On the other hand, if $I$ is a segment and $C$ is convex, the set $I \cap C \backslash \exp C$ is also a segment, so it is $F_{\sigma}$. Therefore $\bigcup\{I \cap C \backslash \exp C ; I \in \mathcal{I}\}$ is $F_{\sigma}$.

The set $E$ can be written as the countable union of sets $E_{n}$ of all elements of $E$ such that the plane $S$ and the segment $[x, c]$ from the definition of $E$ can be found 
so that $\|c-x\| \leq 1$ and the scalar product of $c-x$ and of the unit normal vector to $S$ does not belong to the open interval $\left(-\frac{1}{n}, \frac{1}{n}\right)$. Then each $E_{n}$ is a closed set, which concludes the proof.

Due to Lemma 6.1 it is enough to prove the following proposition to conclude the proof of Theorem 4.2

Proposition 6.2. Let $C$ be a convex $\Sigma_{\alpha}^{0}$ subset of $\mathbb{R}^{3}$. Then the set

$$
U^{C}=\bigcup\{[a, b] ;[a, b] \subset C \cap \partial C\}
$$

is a $\Sigma_{\alpha}^{0}$ set in $\mathbb{R}^{3}$.

Let us realize that we know that $\bigcup\{] a, b[;] a, b[\subset C \cap \partial C\}$ is in $\Sigma_{\alpha}^{0}$ from (the proof of) Theorem 4.1. The point distinguishing the case of exposed points from the case of extreme points is that we need to include the endpoints in our description, which makes it more delicate (recall that in the proof of Example 3.1 the set $S$ is the union of open segments in $\mathbb{R}^{3}$, it is a $G_{\delta}$ set but, if we take the union of the closed segments, we get a set which can be in an arbitrarily high Borel class). We are going to describe $U^{C}$ as the union of countably many sets, which will be studied separately.

It will be useful to note that $U^{C}$ can also be written as

$$
U^{C}=\{x \in C ; \exists c \in C,[x, c] \subset \partial C\} .
$$

We call a subset $D$ of $\mathbb{R}^{3}$ a rational disc if it is a closed disc with positive rational radius in a plane $P \subset \mathbb{R}^{3}, P$ is perpendicular to one of the axes and the center of $D$ has rational coordinates. Let $\mathcal{D}$ denote the countable set of all rational discs.

For a fixed rational disc $D$ in a plane $P$ we define the multivalued map $\Phi_{D}$ taking points of $\mathbb{R}^{3} \backslash P$ to compact subsets of $D$ by

$$
\Phi_{D}(x)=\{y \in D ;[x, y] \subset \partial C\},
$$

and we put

$$
S_{D}=\left\{x \in \mathbb{R}^{3} \backslash P ; \Phi_{D}(x) \text { is a singleton }\right\} .
$$

By restricting $\Phi_{D}$ to $S_{D}$, and identifying the singletons $\Phi_{D}(x)$ with their elements, we define the function $\varphi_{D}: S_{D} \rightarrow D$ such that

$$
\left\{\varphi_{D}(x)\right\}=\Phi_{D}(x) \text { for } x \in S_{D}
$$

Claim 6.3. Let $D \in \mathcal{D}$ be arbitrary and $P \supset D$ be a plane. Then the multivalued map $\Phi_{D}$ is upper semi-continuous (i.e. $\Phi_{D}^{-1}(F)=\left\{x ; \Phi_{D}(x) \cap F \neq \emptyset\right\}$ is closed in $\mathbb{R}^{3} \backslash P$ for every closed $\left.F \subset D\right)$ and the set $S_{D}$ is $G_{\delta}$.

In particular, we get that $\Phi_{D}^{-1}(F) \in F_{\sigma}$ if $F \in F_{\sigma}$ and the restriction $\varphi_{D}: S_{D} \rightarrow$ $D$ of $\Phi_{D}$ to $S_{D}$ is continuous.

Proof. The first claim follows since $\partial C$ is closed and the disc $D$ is compact. For the other one it is enough to realize that the sets $F_{n}=\left\{x \in \mathbb{R}^{3} \backslash P\right.$; diam $\left.\Phi_{D}(x) \geq \frac{1}{n}\right\}$ are closed in $\mathbb{R}^{3} \backslash P$ for $n \in \mathbb{N}$, the set $F=\left\{x \in \mathbb{R}^{3} \backslash P ; \Phi_{D}(x) \neq \emptyset\right\}$ is closed in $\mathbb{R}^{3} \backslash P$ and $S_{D}=F \backslash \bigcup_{n \in \mathbb{N}} F_{n}$.

Let

and

$$
U_{f}=\bigcup\{F ; F \text { is a two-dimensional face of } \bar{C}\}
$$

$U_{s}=\{x \in \partial C ;$ there are at least three disjoint segments $] a_{i}, x\left[\subset \partial C \backslash U_{f}\right\}$. 
Claim 6.4. We have the equalities

$$
U^{C}=C \cap \bigcup_{D \in \mathcal{D}} \Phi_{D}^{-1}(C \cap D)=\left(C \cap \bigcup_{D \in \mathcal{D}} \varphi_{D}^{-1}(C \cap D)\right) \cup\left(U_{f} \cap U^{C}\right) \cup\left(U_{s} \cap U^{C}\right) .
$$

Proof. The first equality and the inclusion

$$
U^{C} \supset\left(C \cap \bigcup_{D \in \mathcal{D}} \varphi_{D}^{-1}(C \cap D)\right) \cup\left(U_{f} \cap U^{C}\right) \cup\left(U_{s} \cap U^{C}\right)
$$

are clear.

Let $x \in U^{C} \backslash\left(U_{f} \cup U_{s}\right)$. Then $[c, x] \subset C \cap \partial C$ for some $c$. There is a rational disc $D$ with $D \cap] c, x$ [ being a singleton and $D$ does not meet any segment $] c^{\prime}, x\left[\subset \partial C \backslash U_{f}\right.$ such that $] c^{\prime}, x[\cap] c, x\left[=\emptyset\right.$. Since $x \notin U_{f}$, there is no segment $\left[c^{\prime \prime}, x\right] \subset C \cap \partial C \cap U_{f}$. By noting that for any segment $] a, b[\subset \partial C$ either $] a, b\left[\subset \partial C \backslash U_{f}\right.$ or $[a, b] \subset U_{f}$, this implies that $x \in S_{D}$ and $\varphi_{D}(x)$ is the only element of $] c, x[\cap D$. Since $] x, c[\subset C$ we get that $\varphi_{D}(x) \in C \cap D$, so $x \in \varphi_{D}^{-1}(C \cap D)$.

Claim 6.5. The set $U_{f} \cap U^{C}$ is relatively $F_{\sigma}$ in $C$.

Proof. Note first that there are countably many two-dimensional faces $F_{k}, k \in \mathbb{N}$, of $\bar{C}$. Let $N_{i}=\left\{k \in \mathbb{N} ; F_{k} \cap C\right.$ is an $i$-dimensional face of $\left.C\right\}$ for $i=0,1,2$. Then

$$
\bigcup_{k \in N_{1} \cup N_{2}} F_{k} \cap C \subset U_{f} \cap U^{C} \subset \bigcup_{k \in N_{0} \cup N_{1} \cup N_{2}} F_{k} \cap C .
$$

Since $\bigcup_{k \in N_{0}} F_{k} \cap C$ is a countable set and each $F_{k} \cap C$ is relatively closed in $C$, this implies that $U_{f} \cap U^{C}$ is $F_{\sigma}$ in $C$.

Lemma 6.6. Let $K$ be a closed convex set, and let $U_{f}$ denote the union of the two-dimensional faces of $K$. Then the set $U_{s}$ of points $x$ for which there exist three pairwise disjoint segments $] x, b_{1}[] x,, b_{2}[] x,, b_{3}\left[\subset \partial K \backslash U_{f}\right.$ is countable.

Proof. First we claim that an open segment in $\partial K \backslash U_{f}$ cannot intersect any nonparallel closed segment of $\partial K$. Indeed, otherwise they would belong to a common supporting plane $S$ of $K$ and $S \cap K$ would be a two-dimensional face of $K$; so both segments would be contained in $U_{f}$.

For each point $x \in U_{s}$ take three pairwise disjoint segments $] x, b_{1}[] x,, b_{2}[] x,, b_{3}[\subset$ $\partial K \backslash U_{f}$, put $a_{i}=\left(x+b_{i}\right) / 2$ and assign the "spider" $S_{x}=\bigcup_{i=1}^{3}\left[x, a_{i}[\right.$ to $x$.

First we show that the spiders $S_{x}, x \in U_{s}$, are pairwise disjoint. Suppose that $x \neq x^{\prime}$ and $y \in S_{x} \cap S_{x^{\prime}}$. We can suppose that $y \neq x$. Then $\left.y \in\right] x, a_{i}\left[\cap\left[x^{\prime}, a_{j}^{\prime}\right]\right.$ for some $i, j$. According to our first observation this may happen only if $] x, a_{i}$ [ and $] x^{\prime}, a_{j}^{\prime}$ [ lie in the same line. Then we must have $\left.x \in\right] x^{\prime}, b_{j}^{\prime}\left[\right.$ or $\left.x^{\prime} \in\right] x, b_{i}[$. The assumption $y \neq x$ was used only to get the last statement that is symmetrical with respect to $x$ and $x^{\prime}$. So we may and shall suppose now that $\left.x \in\right] x^{\prime}, b_{j}^{\prime}[$. Then for at least one $k \in\{1,2,3\}$ the intervals $] x^{\prime}, b_{j}^{\prime}$ [ and $\left[x, b_{k}\right]$ are non-parallel non-disjoint segments in $\partial K \backslash U_{f}$, contradicting our first observation.

One can easily write $\partial K$ as a countable union of relatively open (in $\partial K$ ) sets $T_{n}$ such that each $T_{n}$ can be projected to a plane by an injective projection. By taking the spiders smaller if necessary, we can suppose that each spider is contained in one of the $T_{n}$ 's. Since the projections are injective and the spiders are pairwise disjoint, we get that the projections of the spiders contained in a fixed $T_{n}$ are also pairwise disjoint. 
By the injectivity, the projection of a spider is of the form $\bigcup_{i=1}^{3}\left[y, c_{i}[\right.$, where ]$y, c_{1}[] y,, c_{2}[$ and $] y, c_{3}[$ are pairwise disjoint open segments. We call such plane sets "plane-spiders" for a while.

Therefore what remains to prove is the (probably well-known) fact that there can be only countably many pairwise disjoint plane-spiders in a fixed plane. For proving this, assign to each plane-spider a triangle such that the "head" of the spider is inside the triangle, each "leg" intersects one of the sides of the triangle and all coordinates of all vertices of the triangle are rational.

The observation that to two disjoint plane-spiders distinct "rational" triangles are assigned concludes the proof.

Proof of Proposition 6.2. By the first equality of Claim 6.4 and the upper semicontinuity of the multivalued map $\Phi_{D}$ (Claim 6.3), we have that for $\alpha=2$ the set $U^{C}$ is indeed $F_{\sigma}$.

The $\alpha=1$ case is obvious, so let $\alpha>2$. Then by Claim 6.5, $U_{f} \cap U^{C}$ is in $\Sigma_{\alpha}^{0}$. Applying Lemma 6.6 for $K=\bar{C}$, we get that $U_{s}$ is countable, so $U^{C} \cap U_{s} \in F_{\sigma} \subset \Sigma_{\alpha}^{0}$. By the continuity of $\varphi_{D}$ (Claim 6.3), the set $\varphi_{D}^{-1}(C \cap D)$ is $\Sigma_{\alpha}^{0}$ in $S_{D}$. Since (by Claim 6.3) the set $S_{D}$ is $G_{\delta}$ and $\alpha>2$, this means that $\varphi_{D}^{-1}(C \cap D)$ is in $\Sigma_{\alpha}^{0}$ in $\mathbb{R}^{3}$, too. Therefore, by Claim 6.4 we get that $U^{C}$ is indeed in $\Sigma_{\alpha}^{0}$.

Let us recall that Lemma 6.1 and Proposition 6.2 imply Theorem 4.2 .

\section{REFERENCES}

[1] G. Choquet, H. Corson and V. Klee, Exposed points of convex sets, Pacific Journal of Mathematics 17 (1966), 33-43. MR0198176 (33:6335)

[2] H. H. Corson, A compact convex set in $E^{3}$ whose exposed points are of the first category, Proc. Amer. Math. Soc. 16 (1965), 1015-1021. MR0180917 (31:5147)

[3] P. Holický and V. Komínek, Two remarks on the structure of sets of exposed and extreme points, Extracta Math. 15 (2000), 547-561. MR.1826133 (2001k:46021)

[4] P. Holický and M. Laczkovich, On the descriptive properties of the set of exposed points of a compact convex subset of $\mathbb{R}^{3}$, Proc. Amer. Math. Soc., to appear.

[5] J. E. Jayne and C. A. Rogers, The extremal structure of convex sets, Journal of Functional Analysis 26 (1977), 251-288. MR0511800 (58:23515)

[6] A. S. Kechris, Classical Descriptive Set Theory, Graduate Texts in Mathematics 156, Springer-Verlag, New York 1995. MR1321597|(96e:03057)

[7] K. Kuratowski, Topology, Vol I, Academic Press, New York 1966. MR0217751 (36:840)

[8] R. T. Rockafellar, Convex Analysis, Princeton University Press, Princeton, New Jersey, 1970. MR0274683 (43:445)

[9] C. A. Rogers, The convex generation of convex Borel sets in Euclidean space, Pacific J. Math. 35 (1970), 773-782. MR0278189(43:3920)

Department of Mathematical Analysis, Charles University, Sokolovská 83, 18600 Prague 8, Czech Republic

E-mail address: holicky@karlin.mff.cuni.cz

Department of Analysis, Eötvös Loránd University, PÁzmány Péter sétány 1/C, BuDAPEST, 1117, HUNGARY

E-mail address: elek@cs.elte.hu 